\title{
Kernos
}

Revue internationale et pluridisciplinaire de religion grecque antique

5 | 1992

Varia

\section{À propos de l'iconographie « canonique » d'Isis et des femmes vouées à son culte}

\section{Michel Malaise}

\section{(2) OpenEdition \\ Journals}

\section{Édition électronique}

URL : http://journals.openedition.org/kernos/1069

DOI : 10.4000/kernos.1069

ISSN : 2034-7871

\section{Éditeur}

Centre international d'étude de la religion grecque antique

\section{Édition imprimée}

Date de publication : 1 janvier 1992

ISSN : 0776-3824

\section{Référence électronique}

Michel Malaise, «À propos de l'iconographie " canonique » d'Isis et des femmes vouées à son culte», Kernos [En ligne], 5 | 1992, mis en ligne le 19 avril 2011, consulté le 21 septembre 2020. URL : http:// journals.openedition.org/kernos/1069; DOI : https://doi.org/10.4000/kernos.1069 
Kernos, 5 (1922), p. 329-361.

\title{
REVUE DES LIVRES
}

\section{Compte rendu critique}

\section{À PROPOS DE L'ICONOGRAPHIE «CANONIQUE» D'ISIS ET DES FEMMES VOUÉES À SON CULTE}

\author{
par Michel MALAISE
}

Si l'iconographie de Sérapis a déjà suscité des monographies substantielles, fondées sur une très riche documentation, ou des inventaires ${ }^{1}$, et engendré de nombreux débats ${ }^{2}$, l'étude de l'imagerie d'Isis, de façon assez paradoxale, n'a pas profité des mêmes efforts ${ }^{3}$, peut-être en raison de son caractère plus disparate, reflet d'une nature elle-même plus complexe. En outre, si les sources littéraires nous renseignent sur la création de la statue cultuelle canonique de Sérapis, nous ignorons tout de l'hellénisation de l'image de son épouse. La publication récente de la thèse de Johannes EINGARTNER, Isis und ihre Dienerinnen in der Kunst der römischen Kaiserzeit (Leyde - New York - Copenhague Cologne, E. J. Brill, 1991, (Supplements to Mnemosyne, 115), $24 \times 16 \mathrm{~cm}$, XIII-198 p., 98 pl., 1 ill., 140 fl., ISBN 90-0409312-5) est donc la bienvenue

1 Cf. J.E. Stambaugh, Sarapis under the Early Ptolemies, Leyde, 1972 (EPRO, 25); W. HoRnBosTel, Sarapis. Studien zur Überlieferungsgeschichte, den Erscheinungsformen und Wandlungen der Gestalt eines Gottes, Leyde, 1973 (EPRO, 32), 1973; G.J. KATER-SibBES, Preliminary Catalogue of Sarapis Monuments, Leyde, 1973 (EPRO, 36); V. Tran Tam Tinh, Sérapis debout. Corpus des monuments de Sérapis debout et étude iconographique, Leyde, 1983 (EPRO, 94).

2 Cf. la bibliographie dans V. Tran TAM Tinh, Etat des études iconographiques relatives à Isis, Serapis et Sunnaoi Theoi, in Aufstieg und Niedergang der römischen Welt, II, 17.3, Berlin, New York, 1984, p. 1713-1722, 1734-1735.

3 Mis à part des inventaires partiels, le seul catalogue méthodique paru est réservé à Isis courotrophe : V. TRAN TAM TINH, Y. LABRECQUE, Isis lactans. Corpus des monuments gréco-romains d'Isis allaitant Harpocrate, Leyde, 1973 (EPRO, 37) et V. Tran TAM TINH, De nouveau Isis lactans (Supplément I), in Hommages à M. J. Vermaseren, III, Leyde, 1978 (EPRO, 68), p. 1231-1268. Le chercheur peut aussi se faire une idée de la riche iconographie d'Isis grâce à V. TRAN TAM TINH, art. Isis, in Lexicon iconographicum mythologiae classicae (cité dans la suite LIMC), V, 2, 1990, p. 761-796 et pl. 501-526, contribution que J. Eingartner n'a a pu encore signaler. On trouvera un aperçu des problèmes posés par l'iconographie d'Isis dans l'ouvrage cité à la note 2, p. 1722-1730 et p. 1736. 
puisqu'elle s'attache à l'étude du type iconographique le plus fréquent d'Isis, type que l'on pourrait qualifier avec Tran Tam Tinh de "canonique», et qu'ont repris pour leur compte de nombreuses femmes vouées à son culte.

Le riche matériel ici étudié est constitué de statues, de bustes, de reliefs et de quelques peintures, dont la quasi totalité est d'époque impériale. Le travail est divisé en deux grandes parties: la première aborde l'étude iconographique des représentations d'Isis (Ikonographie und Kunstgeschichte der Isisbilder, p. 8-66); la seconde s'attache à celle des femmes adonnées au culte d'Isis et à la recherche du sens de cette identification par l'image (Ikonographie und Deutung der Isisdienerinnen, p. 67-107). L'ouvrage embrasse donc une double perspective: une enquête de nature proprement iconographique, mais aussi une recherche plus sociologique, qui essaie de déterminer la place réservée au sein du culte aux isiacae ainsi vêtues, en s'aidant pour se faire des sources littéraires et épigraphiques. L'exposé est suivi d'un catalogue de 157 entrées (p. 109175), très richement illustré par 98 planches d'excellente qualité.

Cet ouvrage constitue une importante contribution à l'iconographie isiaque et un précieux recueil de documents. Son auteur nous semble particulièrement bien informé des problèmes et de la bibliographie proprement archéologiques, mais sur le terrain de l'histoire religieuse son information est parfois lacunaire, et nombre d'études récentes auraient dû figurer dans ce travail, même si l'on met à part l'étude de E.J. WALTERS, Attic Grave Reliefs that represent Women in the Dress of Isis, 1988 (= Suppl. 22 à Hesperia) que l'auteur nous dit n'avoir pu intégrer à son manuscrit ${ }^{4}$. Il nous informe toutefois dans sa préface qu'il est arrivé sur cette question à des conclusions concordant pour l'essentiel à celles de son collègue.

Toutes ces raisons nous ont poussé à consacrer à ce livre non un simple compte rendu mais plutôt une chronique bibliographique.

4 Nous aurions aussi beaucoup souhaité intégrer au présent article les conclusions de Walters, mais il ne nous a pas été possible de mettre la main sur cet ouvrage dans les délais dont nous disposions. Nous reviendrons éventuellement dans un prochain numéro de cette revue sur le contenu de cette étude. 


\section{Les statues d'Isis}

Le type "canonique" représente Isis debout, le poids du corps portant sur une seule jambe, brandissant un sistre dans la main droite levée et tenant une situle dans la main gauche abaissée. Cette typologie peut se subdiviser en cinq groupes qui sont fonction des vêtements de la déesse. L'apparition de ces différences vestimentaires, comme le souligne l'auteur, est conditionnée par des circonstances géographiques et chronologiques et ne reflète en rien une fonction spécifique ou un trait mythologique.

L'iconographie de l'Isis "canonique" est dominée par deux toilettes: le type «Knotenpalla» et le type "Diplax». Les statues divines de la première catégorie ( ${ }^{\circ} 9-39$ du catalogue) sont caractérisées par le port d'un long chiton sur lequel est jetée une palla, le plus souvent frangée, nouée entre les seins, pour former le célèbre "nœud isiaque». Ce vêtement, courant dans les reliefs égyptiens de Basse Époque, passe dans la statuaire, dès le début de l'époque ptolémaïque, sur le dos de déesses, de reines, ou de femmes.

Dans la mesure où Isis et ses dévotes, ou à tout le moins certaines d'entre elles, pouvaient porter le même costume, se pose la difficile et irritante question de distinguer la déesse des isiaques. Pour Eingartner, il convient d'attribuer à de simples mortelles les représentations qui figurent sur les monuments funéraires, tout comme les bustes et statues dont les têtes présentent des traits individualisés propres à un portrait. Ce parti-pris paraît raisonnable ${ }^{5}$. Dans le cas des autres œuvres en ronde-bosse, les isiaques se démarqueraient, de façon générale, des Isis par un chiton à manches longues, et par l'absence de voile ou d'emblème sur la tête ${ }^{6}$; en outre, dans la série des œuvres vêtues

5 Exception est faite pour un autel romain (Louvre MA $1544=\mathrm{n}^{\circ} 50$ ) dont l'inscription (CIL, VI, 345 = VIDMAN, Sylloge incriptionum religionis Isiacae et Sarapiacae, Berlin, 1969 ( $R G V V, 28$ ) (cité dans la suite SIRIS), $\mathrm{n}^{\circ} 387$ ) mentionne comme seul dédicant un homme.

6 Feraient exception deux autels funéraires provenant de Rome ( $n^{\circ}$ 132: un uraeus entre deux épis et 135: un croissant de lune avec un uraeus, mais il s'agit dans les deux cas de la représentation de mélanéphores qui ont pu, comme le pense Eingartner, bénéficier de ce privilège en fonction de leur rang supérieur à celui des autres isiaques. En terre égyptienne, on notera sur un cerceuil égyptien en bois d'époque romaine (cf. Kleopatra. Ägypten um die Zeitwende, Mayence, 1989, p.295-296 et fig.) la présence de deux plumes sur la tête d'une isiaque, sans doute une prêtresse, figurée en compagnie de deux prêtres. Par ailleurs, le célèbre décret de Canope (ligne $33=$ Urk. II, 150 et G. ROEDER, Kulte 
d'une "Knotenpalla", le nœud isiaque des mortelles serait toujours constitué des deux extrémités de la palla. Sur les statues d'Isis, comme le remarque Eingartner (p. 13), le nœud est le plus souvent formé à l'aide d'une seule extrémité de l'étoffe tombant des épaules, mais il est parfois noué avec les deux extrémités de ce vêtement. ${ }^{7}$ Cette double possibilité rendrait caduque une intéressante hypothèse formulée par V. Tran Tam $\operatorname{Tinh}^{8}$, ignorée de notre auteur, selon laquelle le nœud symétrique distinguerait les isiaques de leur divine patronne. Ce dernier point est donc d'importance dans l'iconographie isiaque. Quand on examine la documentation réunie par Eingartner, on constate que, seules, six statues et deux bustes attribués par cet auteur à Isis présentent une "Knotenpalla" dont les deux pans servent à former le nœud. Ces huit cas constituent-ils des entorses à une tendance générale ou bien ces œuvres représentent-elles en fait des isiaques et non leur patronne, comme Tran Tam Tinh l'avait suggéré pour l'Isis dite du Capitole $\left(\mathrm{n}^{\circ} 9\right)$ ? Il est difficile de se prononcer pour les statues $\mathrm{n}^{\circ} 10$ (Zagreb, Mus. Arch., 34) et 29 (El-Djem, 3328), ainsi que pour le buste $\mathrm{n}^{\circ} 75$ (Cracovie, Mus. National, $\mathrm{Cz} 1877$ ), qui sont acéphales; mais le voile qui recouvre la tête des statues n ${ }^{\circ} 9,28$ (Rome, Musée du Capitole, 744 et 711), et 27 (Munich, Glyptothèque, 250), tout comme le haut diadème avec disque et emblèmes isiaques de la statue $\mathrm{n}^{\circ} 17$ (Tunis, Bardo, C 982) et le croissant de lune du buste $\mathrm{n}^{\circ} 78$ (Constantza, Mus. Arch., 2002) indiqueraient que les monuments en cause sont bien ceux d'Isis ellemême et que le nœud de la déesse peut donc être agencé de deux manières différentes.

und Orakel im alten Ägypten, Zurich, 1960, p.164) rapporte que les jeunes musiciennes sacrées qui chantent en l'honneur de Bérénice II portent les couronnes des divinités auxquelles elles sont attachées.

7 Dans l'hypothèse d'Eingartner, le nœud est respectivement constitué par les deux extrémités du manteau retombant symétriquement sur les épaules et son ourlet barrant la poitrine, ou par l'extrémité du manteau tombant sur l'épaule droite et la bordure supérieure de son pan transversal. Si l'on accepte l'hypothèse de Bianchi, le nœud symétrique ou asymétrique serait formé par le bord supérieur de la robe et une ou deux extrémités du châle.

8 Cf. V. TRAN TAM TINH, Le culte des divinités orientales en Campanie, Leyde, 1972 (EPRO, 27), p. 31, n. 3; ID., Isis-Nymphe de Laodicée, in Mélanges d'études anciennes offerts à $M$. Lebel, Québec, 1980, p. 345, n. 26; ID., étude citée supra, n. 2, p. 1726-1727; ID., art. Isis, in LIMC, V, p. 792 
L'origine égyptienne du vêtement étudié a été soulignée récemment par R.S. Bianchi ${ }^{9}$ dans un travail qu'ignore malheureusement J. Eingartner. Pour l'auteur de cet article, l'Isis à la «Knotenpalla» n'est pas vêtue, comme on le pensait depuis Schäfer ${ }^{10}$, de deux pièces, mais bien d'un costume tripartite comprenant une tunique montant jusqu'au cou, une robe enroulée autour du corps et s'arrêtant sous les seins, et, sur les épaules, un long châle noué à la robe par un nœud sur le sein droit ${ }^{11}$. En outre, Bianchi montre que, du moins dans les documents proprement égyptiens, le nœud dit «isiaque» n'est pas nécessairement lié à Isis, comme le prouvent trois statues de femmes portant le vêtement tripartite noué sur la poitrine que leurs inscriptions identifient à des joueuses de sistres (ihy.t) attachées aux cultes d'Amon et d'Hathor ${ }^{12}$.

Avant d'aborder les Isis à la "Knotenpalla» de type canonique, Eingartner (p. 10-11; catalogue $n^{\circ} 1-8,11$ ) considère quelques variantes qui s'écartent du type habituel par la position des bras ou par les attributs. On trouve notamment une Isis saisissant un long sceptre de sa main droite levée $\left(n^{\circ} 1-4\right)$ ou avancée $\left(n^{\circ} 11\right)$, ou le maintenant de sa main gauche abaissée $\left(n^{\circ} 5-7^{13}\right)$. À ce dernier type, il faudrait sans doute rattacher également une statue provenant du Sérapeum $C$ de Délos ${ }^{14}$. On notera que la statue $\mathrm{n}^{\circ} 6$ (Vatican, Museo Chiaramonti, 1717) n'est pas en fait une Isis mais bien, comme l'a montré récemment J.-Cl. Grenier ${ }^{15}$, une image de Cléopâtre VII, en Isis, se suicidant. Le fort déhanchement, la tête tournée vers la droite et le bras droit replié vers le sein font déjà hésiter à y reconnaître une Isis, mais tout doute disparaît quand on constate la présence, discrète il est vrai, d'un petit

9 Cf. R.S. BIANCHI, Not the Isis Knot, in Bulletin of the Egyptological Seminar, 2 (1980), p. 9-31.

10 Cf. H. SCHÄFER, Das Gewand der Isis, in Janus, 1 (1921), p. 194-206.

11 Cette manière de draper, puis de nouer robe et châle a fait l'objet d'expériences réelles.

12 Cf. BIANCHI, op. cit., p. 18-19 qui cite trois statues inédites du Musée du Caire (JE 37027; JE 38017; JE 37453).

13 À propos de l'Isis figurant sur le relief de Rhodes ( $\left.n^{\circ} 7\right)$, V. TRAN TAM TINH, étude citée supra, n. 2, p. 1723 assigne comme patrie à ce type, vu son empreinte hellénistique nette, la ville d'Alexandrie.

14 Cf. V. Tran Tam Tinh, art. Isis, in $L I M C$, V, 2, p. 765, n 21; J. MarCadÉ, $A u$ Musée de Délos, Paris, 1969, p. 430 et pl. 57.

15 Cf. J.-Cl. Grenier, Notes isiaques I, Monumenti Musei e Gallerie Pontificie, 1989, (extrait du Bolletino, IX,1), p.10-16 et fig. 4-6. 
serpent qui ondule sur le sein droit. On ajoutera à la notice du catalogue d'Eingartner que cette sculpture est réputée provenir de la Villa Adriana. Atypique aussi est une statuette hellénistique de Turin $\left(\mathrm{n}^{\circ} 8=\right.$ Mus. di Antichità, 673) vêtue seulement d'une «Knotenpalla», et dont la tête et les bras, plus animés que de coutume, manifestent peut-être la douleur éprouvée par Isis dans la quête de son époux défunt. À ces variantes, il conviendrait d'ajouter une statue en marbre de Berlin (Staatliche Mus., 12440) d'Isis portant, suivant l'habitude, la situle dans la main gauche abaissée, mais serrant de manière inhabituelle un serpent dans la main gauche avancée vers le spectateur ${ }^{16}$; la coiffure est tout aussi originale : elle est faite d'un uraeus se détachant d'un disque solaire derrière lequel surgissent trois épis.

Parmi les nombreuses copies romaines (statues $n^{\circ} 9-39$ et bustes $n^{\circ}$ 74-79) du type à la «Knotenpalla»,- qu'Eingartner présente dans un ordre chronologique fondé sur une étude stylistique, tout particulièrement du vêtement, - l'exemple le plus ancien, mais aussi le plus connu, est fourni par l'Isis du Capitole (Inv. $744=\mathrm{n}^{\circ} 9$ ). La draperie qui couvre le torse de cette statue, que son style permet de dater des années 40-50 de notre ère, est nouée entre les deux seins; la palla présente un arrangement symétrique des plis qui, depuis la poitrine, retombent de part et d'autre de l'axe du corps dont le vêtement collant laisse transparaître les formes. La chevelure aux caractéristiques boucles «libyennes", alexandrines avant d'être isiaques, est couverte d'un voile que l'on retrouve sur de nombreuses autres statues d'Isis, mais repérable, tout au plus, sur la tête d'une seule isiaque ${ }^{17}$. La datation assez haute de cette statue à l'intérieur de l'époque impériale et la présence de ce voile sur d'autres Isis de la fin du Ier siècle ( $n^{\circ} 80$ et 93 ) rendent caduque l'hypothèse émise par Tran Tam Tinh ${ }^{18}$, selon laquelle cet élément n'apparaîtrait pas avant les Antonins et serait imputable au goût égyptisant d'Hadrien, qui aurait conduit «à romaniser le klaft égyptien réintroduit dans l'art religieux isiaque».

16 Cf. S. Wenig, Die Frau im alten Agypten, Leipzig, 1967, p. 53, nº 107 et pl.

17 Il s'agit de l'autel funéraire romain de Cantinea Procla datable des années 7080 de notre ère (cf. p. 79 et $\mathrm{n}^{\circ}$ 132). Il faut toutefois convenir que l'on ne peut exclure que la figure féminine qui orne la face principale du monument, audessus de l'inscription, soit en réalité la déesse Isis elle-même, d'autant plus que le voile est surmonté d'un uraeus entre deux épis, emblème exceptionnel pour une mortelle.

18 Cf. V. Tran TAM Tinh, Le culte des divinités orientales en Campanie, p. 33-34; ID., in Mélanges Lebel, p. 345. Cette hypothèse a échappé à Eingartner. 
Cette image «canonique» de l'Isis à la «Knotenpalla» s'introduit donc lentement durant le Ier siècle, puis se répand de plus en plus à partir de l'époque de Hadrien, pour s'éteindre à la fin du IIIe siècle. Si la forme du nœud, d'abord du type "knopfartig», se transforme ensuite en un plus gros nœud coulant dont l'extrémité pend vers le bas, et si le traitement du vêtement subit des modifications sous le règne d'Hadrien et au début de l'époque antonine, les caractéristiques présentées par l'Isis du Capitole en cette matière se retrouveront encore au IIe siècle. Selon Eingartner, pareille persistance indique que ces éléments faisaient partie de la statue de culte originale. Ce prototype, d'abord situé au tournant du IVe-IIIe siècle av. J.-C., est aujourd'hui, en général, regardé comme une création d'époque romaine. Pour Eingartner, la combinaison raffinée des éléments du vêtement, certains plus raides, d'autres plus libres, rend peu concevable de situer le prototype avant le milieu du IIe siècle av. J.-C. Il s'agirait d'une création éclectique, redevable à l'Égypte du choix du vêtement et de sa disposition sage et axiale, mais tributaire de l'art hellénistique pour le traitement des plis. Pareille création aurait vu le jour dans le monde grec, et sans doute à Athènes, d'où proviennent la plupart des stèles représentant des isiaques revêtues des mêmes atours. La date avancée serait confirmée par l'image d'une isiaque en "Knotenpalla» sur une stèle de Smyrne $\left(\mathrm{n}^{\circ} 99\right)$ que des critères épigraphiques permettent de faire remonter aux alentours de l'an 100 av. J.-C. L'origine non égyptienne de la statue originale semble trouver un appui dans certaines remarques faites par Tran Tam Tinh, mais non invoquées par Eingartner. Tran Tam Tinh ${ }^{19}$ observe que le type à la "Knotenpalla" est presque absent de la statuaire en Égypte (cf. l'Isis de Ras el Soda ${ }^{20}$ et peut-être celle du petit Sérapéum de Louxor); qu'il fait défaut dans la numismatique alexandrine et est plutôt rare dans le monde des terres cuites romanoégyptiennes. On ajoutera que, d'une façon générale, il est encore assez peu répandu au Ier siècle de notre ère, comme l'indique son absence à Délos, à Pompéi, à Herculanum et à Alexandrie. Tran Tam Tinh, qui propose pour l'Isis du Capitole une date plus basse, le IIe s. ap. J.-C.,

19 Cf. V. TRAN TAM TINH, in Aufstieg und Niedergang der römischen Welt, II, 17.3, p. 1725-1727; ID., art. Isis, in LIMC, V, p. 792.

20 Cf. V. Tran TAM Tinh, art Isis, in LIMC, V, p. 764, n 9; F. Le Corsu, Isis. Mythe et mystères, Paris, 1977, p. 216-219 et fig.; F. Dunand, Le culte d'Isis dans le bassin oriental de la Méditerranée, I, Leyde, 1973 (EPRO, 26), pl. XI, 1. Cette statue, dont l'avant-bras droit cassé devait tenir un serpent, n'est pas mentionnée dans l'ouvrage d'Eingartner. 
pense même que ce type fut seulement créé vers la fin du Ier s. ou au début du IIe s. ap. J.-C., peut-être "pour remplacer l'uraeus (que tenait la déesse) par le sistre à cause de la difficulté de faire passer le message symbolique du cobra dans des milieux culturels non égyptiens» 21 .

Pour terminer avec ce type, on ajoutera que la liste d'Eingartner peut être enrichie d'une statue provenant de Sarsina ${ }^{22}$, ainsi que de quatre statues de marbre, à la tête voilée, dont les bras, il est vrai, manquent ${ }^{23}$.

Les statues de type "diplax» ( $\left.n^{\circ} 40-73\right)$ représentent Isis vêtue d'un chiton à manches mi-courtes et d'un manteau plié en deux (diplax), qui, une fois enroulé, avec le pli sous l'aisselle gauche, est attaché sur l'épaule droite, libérant ainsi le bras gauche. La statue de Munich, datable de 30-50 ap. J.-C., constituerait une copie particulièrement fidèle de l'original. Celui-ci serait à nouveau marqué par un éclectisme, où se mêlent dans le traitement du drapé goût hellénistique et penchant pour une certaine raideur, une combinaison qui indiquerait une création à situer aux confins de l'époque hellénistique et impériale. Cette iconographie, limitée à la moitié occidentale de l'empire, aurait été conçue à Rome, d'où proviennent de nombreuses statues d'Isis et quelques représentations d'isiaques. Selon Eingartner, c'est sous le règne de Caligula, après le temps des persécutions dirigées à Rome contre les cultes venus d'Égypte, que ce type aurait vu le jour. Par la suite, il connaît une évolution stylistique comparable à celle de l'Isis à la "Knotenpalla» et survivra, comme cette dernière, jusque vers la fin du IIIe siècle.

Après avoir examiné les bustes ( $n^{\circ} 74-79$ et 83 ) et les têtes d'Isis ( $n^{\circ}$ 80-82 et 84), l'auteur présente les emblèmes qui peuvent orner la coiffure des Isis étudiées jusqu'ici: un simple bandeau; le basileion ou coiffure hathorique constituée de cornes, du disque solaire et éventuellement de deux plumes; un croissant de lune, qui rapproche Isis de Séléné; ou un haut diadème, sans doute couronne d'Isis Regina ou d'Isis-Junon. Sont parfois associés à ces éléments des épis de blé, des capsules de pavots, un uraeus ou des boutons de lotus.

21 Cf. V. Tran TAM Tinh, art. Isis, in LIMC, V, p. 792.

22 Cf. V. Tran TaM Tinh, Le culte des divinités orientales en Campanie, p. 34, n. o); ID., art. Isis, in LIMC, V, p. 766, $\mathrm{n}^{\circ} 29$; M. MALAISE, Documents nouveaux et points de vues récents sur les cultes isiaques en Italie, in Hommages à M.J. Vermaseren, II, Leyde, 1978 (EPRO, 68), p. 634-5, $\mathrm{n}^{\circ} 2$.

23 Cf. V. Tran Tam Tinh, op. cit., p. 767, n 49 (Salamine de Chypre), 54 (Reina), 56 (Valladolid); p. $768, n^{\circ} 58$ et pl. (Vienne). 
Outre les deux images fréquentes que nous venons d'analyser, il existe d'autres types, mais beaucoup plus rares et qui apparaissent plus tard, seulement vers la fin du Ier ou au début du IIe siècle.

Un premier groupe de statues, malheureusement acéphales, est caractérisé par le port d'une palla contabulata $\left(\mathrm{n}^{\circ} 85-88\right)$; nous reviendrons plus loin sur ces documents qui représentent plutôt des isiaques que leur divine patronne.

Sur les statues d'un second groupe ( $\left.n^{\circ} 89-92\right)$, Isis, coiffée d'un voile, est vêtue d'une «Knotenpalla» et d'un himation, le manteau grec étant jeté sur la palla nouée. L'exemple le plus ancien de cette iconographie, limitée aux provinces occidentales de l'empire romain, provient de Lambèse et date de l'époque d'Hadrien. La tête d'une de ces sculptures $\left(n^{\circ} 92\right)$ supporte un croissant de lune avec uraeus.

Enfin, la déesse, la tête à nouveau voilée, est parfois habillée d'un chiton et d'un himation ( ${ }^{\circ}$ 93-97), qui, dans un mouvement large, couvre le corps en sautoir, de l'épaule droite au flanc gauche. Ce type, que l'on trouve aussi bien dans les provinces orientales qu'occidentales, est inspiré par une adaptation hellénistique tardive, connue sous le nom d'“Euterpe Milet», de l'Héra Campana du début de l'époque hellénistique. L'exemple le plus ancien, retrouvé à Thessalonique, date de la fin du Ier siècle de notre ère. Deux de ces statues portent un emblème sur la tête: trois uraeus devant des épis $\left(n^{\circ} 94\right)$ et un disque entre deux cornes de vache $\left(n^{\circ} 97\right)$.

\section{Les représentations d'isiaques}

Avant d'aborder l'étude des reliefs et des statues des femmes attachées au culte d'Isis, Eingartner essaye de dégager d'emblée la signification d'une tenue empruntée à celle de leur déesse. Pour ce faire, il analyse l'épitaphe de Dionysia de Mégalépolis qui resta au service d'Isis depuis l'âge de 15 ans jusqu'à la mort. L'épigramme, du IIe-IIIe siècle, nous apprend que Dionysia portait les vêtements de la déesse elle-même et que c'est dans ces atours qu'elle fut enterrée ${ }^{24}$. F. Dunand $\mathrm{d}^{25}$ estimait que les nombreuses stèles funéraires attiques figurant des isiaques dans le costume de leur patronne représentaient avant tout des initiées, sans exclure que certaines d'entre elles soient à

24 Ce dernier détail nous est aussi fourni par Plutarque (De Iside, 3), qui explique que le vêtement rituel sert de parure mortuaire aux isiaques.

25 Cf. F. Dunand, Le culte d'Isis dans le bassin oriental de la Méditerranée, II, Leyde, 1973 (EPRO, 26), p. 148-150. 
l'occasion des prêtresses ou des membres d'associations cultuelles vouées à Isis. Eingartner suppose que, de façon générale, les images d'isiaques sont des initiées vouées au culte d'Isis, au sein duquel elles remplissaient des fonctions cultuelles subalternes. À propos du costume des initiés, il aurait fallu rappeler le passage d'Apulée où Isis s'adresse au myste Lucius en ces termes «les ornements de la déesse que tu as revêtus dans ta province doivent rester dans le temple où tu les as déposés. Tu ne peux donc ni à Rome les porter aux jours de fête pour faire tes dévotions, ni, si l'ordre t'en est donné, te montrer dans la splendeur de ce bienheureux costume» 26 . Cette révélation d'Isis montre que les initiés se paraient de leur costume aux grandes occasions. Nous reviendrons, comme Eingartner, sur l'identification exacte de ces femmes, après avoir examiné leurs diverses représentations et leur diffusion géographique, et surtout chronologique.

Tant dans les provinces orientales que dans les provinces occidentales de l'Empire romain, les représentations funéraires d'isiaques commencent avec le début de l'époque impériale, et s'étendent loin dans le IIIe siècle, voire même dans le IVe siècle ${ }^{27}$. Deux documents provenant de Smyrne sont plus anciens. Le premier ${ }^{28}\left(n^{\circ} 98\right)$ des environs de 200 av. J.-C., représente une isiaque avec sistre et situle, attributs alors inconnus entre les mains d'Isis, et vêtue d'un chiton et d'une «Knotenpalla», encore nouée avec une extrémité du manteau, comme sur les statues des princesses ptolémaïques, mais dont le drapé est éloigné de celui observé sur les images d'Isis. Le second ( $\mathrm{n}^{\circ} 99$ ), d'environ 100 av. J.-C., montre une isiaque qui, bien que fort endommagée, semble déjà appartenir à l'iconographie "canonique".

Sur les monuments funéraires du monde grec ( $\left.n^{\circ} 100-129\right)$, et tout spécialement à Athènes, toutes les isiaques portent la "Knotenpalla». Cette iconographie, la mieux attestée, est parfois enrichie d'une guirlande florale, empruntée à Isis, qui barre la poitrine en diagonale. Sur certaines stèles, ces femmes sont accompagnées d'un homme vêtus comme leur compagne et brandissant aussi sistre et situle. De l'opinion d'Eingartner, ces hommes et ces femmes seraient tous des initiés. On

26 ApuleE, XI, 29 ( trad. P. Vallette des "Belles Letttres»); cf. le commentaire de J.G. GrIfFITHS Apuleius of Madauros. The Isis-Book (Metamorphoses, Book $X I$ ), Leyde, 1975 (EPRO, 39), p. 339-340.

27 Cf. les $n^{\circ} 138$ et 139 qui proviennent d'Oxyrhynchos.

28 À la bibliographie indiquée, il convient d'ajouter: G. PETZL, Die Inschriften von Smyrna, I, Bonn, 1982, n 10. 
retrouve ce vêtement sur les épaules de cartonnages de momie de l'époque romaine ( $\left.\mathrm{n}^{\circ} 142-144\right)$, sans doute ici dans le but d'assimiler la défunte à $\operatorname{Isis}^{29}$.

Dans les provinces occidentales, les isiaques sont beaucoup plus rares sur les stèles funéraires ( $n^{\circ} 130-137$ ) et proviennent surtout de Rome et d'Italie. Les types sont aussi beaucoup plus variés qu'en terre grecque. Sur deux stèles romaines $\left(\mathrm{n}^{\circ} 130-131\right)$ du IIe siècle ap. J.-C., la femme est vêtue d'un manteau du type «diplax».

Le costume le plus fréquent est constitué de la palla contabulata $\left(n^{\circ} 132-139^{30}\right)$. La palla, faisant le tour du corps, passe sous le bras droit pour remonter sur l'épaule gauche, d'où son extrémité libre retombe par devant, se terminant en un rang de franges. On retrouve cette palla enroulée sur des peintures campaniennes qui lui attribuent une couleur noire ${ }^{31}$. C'est sans doute elle aussi que décrit Apulée ${ }^{32}$ lorsqu'il dépeint Isis couverte d'un manteau d'un noir intense (palla nigerrima) à la bordure brodée d'étoiles et du croissant lunaire. Parfois, la palla portée en sautoir fait plutôt penser à une étole. Eingartner, s'appuyant sur deux documents ( $n^{\circ} 88$ et 151), estime cependant qu'il s'agit toujours bien d'un manteau plié et enroulé autour du corps. D'autres auteurs ${ }^{33}$ reconnaissent dans certains cas une véritable stola. Comme l'exprime Cl. Traunecker ${ }^{34}$ dans un intéressant article non cité par Eingartner, «Tout se passe comme si le manteau noir ... était remplacé symboliquement par une étole ... drapée comme la pièce de vêtement originale». Cette hypothèse paraît exacte, surtout lorsque l'on observe sur une

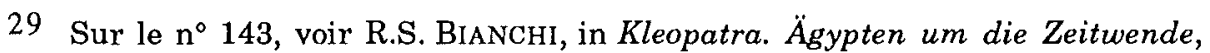
Mayence, $1989 \mathrm{n}^{\circ} 76$ et pl. en couleurs; ID., in Ancient Egyptian Art in the Brooklyn Museum, New York, $1989, \mathrm{n}^{\circ} 83$ et pl. en couleurs.

30 Les statues acéphales $n^{\circ} 85-88$ sont sans doute, elles aussi, des représentations d'isiaques.

31 Cf. les références données par Eingartner, p. 74, n. 417. Il peut s'agir d'isiaques, mais aussi d'Isis.

32 APULEE, XI, 3-4. Sur les difficultés soulevées par ce passage, cf. J.G. GRIFFITHS, Apuleius of Madauros. The Isis-Book, p. 128-132; Cl. Traunecker, L'étole diaconale copte 'et ses antécédents, in Deuxième Journée d'Etudes Coptes, Louvain, Paris, 1986 (Cahiers de la Bibliothèque Copte, 3), p. 102-103.

33 Cf. V. Tran TAM Tinh, Le culte des divinités orientales en Campanie, p. 82, n. 1.

34 Cf. Cl. Traunecker, op. cit., p. 103. 
mosaïque $\mathrm{e}^{35}$ d'Antioche sur l'Oronte, - à laquelle Eingartner ne fait pas référence,- la figure de femme, Isis ou beaucoup plus vraisemblablement une isiaque, participant à un cortège, qui porte clairement une étole en sautoir. La bordure de la palla ou l'étole est parfois brodée de symboles isiaques : couronnes, lune et étoiles ${ }^{36}$.

Le monument le plus ancien attestant l'usage de la palla contabulata est l'autel funéraire ( ${ }^{\circ} 132$ ) érigé à Rome entre 70 et 80 ap. J.-C. pour l'isiaque Cantinea Procla. Ce vêtement noir remonte cependant à l'époque ptolémaïque et devait caractériser l'Isis melanephoros ou melanostolos ${ }^{37}$ des textes, c'est-à-dire une Isis vêtue de noir en signe de deuil pour la mort de son époux Osiris. À cet égard, il conviendrait d'insister sur le fait que la couleur noire comme signe de deuil n'est assurément pas un symbole d'origine égyptienne $\mathrm{e}^{38}$. Pour Eingartner, les isiaques habillées de noir sont à identifier aux mélanéphores des inscriptions ${ }^{39}$; cette hypothèse est vraisemblable, mais il faudrait signaler l'abondante bibliographie consacrée à cette catégorie du personnel isiaque ${ }^{40}$. Dans la mesure où deux ( $n^{\circ} 132$ et 135) d'entre elles

35 Cf. R.E. WITT, Isis in the Graeco-Roman World, Londres, 1971, pl. 35; F. LE CoRsu, Isis. Mythe et mystères, p. 238 et fig.; J.G. GRIFFITHS, Apuleius of Madauros. The Isis-Book, p. 130-131; Cl. TraunECKER, op. cit., p. 103 et fig.15.

36 Cf. les $n^{\circ} 85,86,135$ d'Eingartner; la mosaïque d'Antioche citée supra, n. 35 et la description d'Apulée (XI, 4). Sur la signification de ces astres et des couronnes, voir V. TRAN TAM TINH, Une statuette d'Isis-Ourania, in RA (1970), p. 283-296; J.G. GRIFFITHS, op. cit., p. 130-132; Cl. TRAUNECKER, op. cit., p.103-107.

37 Cf. M.-F. BASLEZ, Recherches sur les conditions de pénétration et de diffusion des religions orientales à Délos (IIe - Ier s. avant notre ère), Paris, 1977, p. 302.

38 Cf. J.G. Griffiths, Apuleius of Madauros. The Isis-Book, p. 128; Cl. Traunecker, op. cit,, p. 105.

39 Dans le domaine épigraphique, les mélanéphores sont attestés à Rome (cf. VIDMAN, SIRIS, $\mathrm{n}^{\circ}$ 426-427) et dans le monde grec à Érétrie (cf. ID., op. cit., $\mathrm{n}^{\circ} 75$ ) et surtout à Délos (cf. P. Roussel, Les cultes égyptiens à Délos du IIIe au ler siècle av. J.-C., Nancy, 1916, $\mathrm{n}^{\circ}$ 21, 26, 58, 58bis, 94, 98, 105, 115, 117, 131, 131bis, 133, 151, 160, 164, 173, 212 (?) et p. 220).

40 Cf. J.G. GrIFFITHS, Apuleius of Madauros. The Isis-Book, p. 128; Ph. BrUNEAU, Le sanctuaire et le culte des divinités égyptiennes à Erétrie, Leyde, 1975 (EPRO, 45), p. 112-114; M.-F. BASLEZ, Une association isiaque: les Mélanéphores, in Chronique d'Egypte, 50 (1975), p. 297-303; EAD., Recherches sur les conditions de pénétration et de diffusion des religions orientales à Délos, p.243-246; H.-B. SCHÖNBORN, Die Pastophoren im Kult der ägyptischen Götter, Meisenheim am Glan, 1976 (Beiträge zur klassischen Philologie, 80), p. 64-69. On ajoutera que la qualité de mélanéphore n'était peut-être pas réservée aux seules personnes vêtues de la palla contabulata ou de l'étole portée en sautoir, car le buste d'un prêtre isiaque retrouvé à Sabratha (cf. G. PESCE, Il tempio 
ont la tête couronnée d'un emblème divin, Eingartner assigne à ces mélanéphores, qui se regroupaient en synodes, une fonction sacerdotale $^{41}$, qui les aurait distinguées des autres isiaques, à considérer elles comme de simples initiées, et à assimiler aux thérapeutes des inscriptions. Cette dernière identification est loin d'être évidente. En effet, comme l'a suggéré Baslez ${ }^{42}$, le mot "thérapeute» semble synonyme de fidèle en général, or tout fidèle n'était évidemment pas initié. Par ailleurs, Eingartner insiste sur le fait que les représentations de mélanéphores sur les monuments funéraires sont réservées à la partie occidentale de l'empire, si l'on met à part deux stèles funéraires ( $\mathrm{n}^{\circ} 138$ 139) provenant de Behnessa-Oxyrhynchos, et deux portraits romains de momie ( $\mathrm{n}^{\circ} 145-146$ ).

Cette limitation, qui serait imputable à une interpretatio romana marquée par l'influence égyptienne, disparaîtrait avec le IIe siècle ap. J.-C. dans le domaine des portraits en ronde bosse $\left(\mathrm{n}^{\circ} 88\right.$ provenant de Tralles en Asie Mineure, $\mathrm{n}^{\circ} 151$ provenant d'Athènes, $\mathrm{n}^{\circ} 156$ de provenance inconnue). Pareillement, la "Knotenpalla" d'abord typique des reliefs funéraires du monde grec, peut revêtir, à partir du début de IIe siècle, les statues d'isiaques ( $\left.n^{\circ} 147-50,152,154-155\right)$, même lorsqu'elles émanent des provinces occidentales ( $n^{\circ} 148-150$ et 151 ).

\section{Identification des isiaques}

Il est bien difficile d'établir avec certitude les fonctions exercées au sein des cultes isiaques par les femmes qui arborent la tenue d'Isis et brandissent ses attributs, sistre et situle.

Pour Eingartner, toutes nos isiaques sont des initiées, étrangères au clergé, à l'exception des mélanéphores. Il constate en effet que les femmes engagées dans l'exercice du culte portaient un autre genre de vêtement. Ainsi, les trois statues de femmes agenouillées retrouvées dans l'Iséum de Bénévent ${ }^{43}$ sont vêtues, la première d'un chiton sur

d'Iside in Sabratha, Rome, 1953, p. 51-52, nº 25 et fig. 31; M.-F. BASLEZ, op. cit., p. 245) est recouvert d'une draperie, dont l'extrémité est rejetée sur l'épaule gauche, qui présente des traces de couleur noire.

41 M.F. BASLEZ, op. cit., p. 244 voit aussi dans les mélanéphores des prêtres de rang inférieur.

42 Cf. M.F. BASLEZ, op. cit., p. 192-195.

43 Cf. H.W. MüLLER, Der Isiskult im antiken Benevent, Berlin, 1969 (MÄS, 16), $\mathrm{n}^{\circ} 285,287,290$ et pl. 31,33-34. Cette position agenouillée est également observée par deux participants, une femme et un homme, qui assistent à la danse sacrée sur une fresque isiaque d'Herculanum (cf. V. Tran TAM TiNH, Le 
lequel sont placés une sorte de châle qui couvre le haut du corps et un manteau drapé maintenu sous la poitrine par un nœud; la seconde d'un chiton, d'un châle noué entre les deux seins et d'un manteau serré autour des hanches; la troisième d'un vêtement d'une seule pièce serré autour du corps sous la poitrine, puis passant sur les deux épaules pour venir se nouer entre les seins. Une des caractéristiques de ces tenues est le manteau qui s'arrête sous les seins ou sur le bassin. Cet agencement rappelle le vêtement d'officiants masculins ${ }^{44}$, une sorte de manteau drapé sous les aisselles ou autour des hanches et descendant jusqu'aux mollets ou aux pieds, offrant ainsi l'allure d'une espèce de long pagne blanc drapé. La femme qui clôture le cortège isiaque du relief Mattei ${ }^{45}$ porte aussi, sur un vêtement de dessous serré sous les seins, un manteau drapé autour des hanches.

Il semble donc bien que les isiaques ne soient pas de véritables prêtresses. Dionysia, qui passa sa vie au service d'Isis et qui fut enterrée dans ses vêtements sacrés n'est désignée dans son épitaphe que par les termes vagues de latris et de propolos. Par ailleurs, F. Dunand ${ }^{46}$ écrivait déjà à propos des nombreuses isiaques des stèles attiques : «Mais il nous paraît aussi difficile de les considérer, toutes, comme des prêtresses; en effet, les stèles qui les représentent s'échelonnent entre la fin du Ier siècle a.C. et la fin du IIe siècle p.C., et la grande majorité d'entre elles sont du IIe siècle; il paraît impossible qu'il y ait eu à Athènes, au IIe siècle, un si grand nombre de 'prêtresses d'Isis', alors que deux sanctuaires seulement accueillaient les dieux égyptiens et que dans l'un d'eux les desservants étaient soumis à une sorte de cumul des fonctions».

S'agit-il pour autant toujours d'initiées ? Le problème est d'autant plus délicat que, selon Eingartner, les deux isiaques représentées sur les stèle de Smyrne ( $\mathrm{n}^{\circ}$ 98-99), respectivement des environs de 200 et de 100 av. J.-C., témoigneraient du désir déjà éprouvé par les initiées de

culte des divinités orientales à Herculanum, Leyde, 1971 (EPRO, 17), pl. XXVIII).

44 Cf. V. Tran Tam Tinh, Essai sur le culte d'Isis à Pompéi, Paris, 1964, pl. IV, 2; ID., Le culte des divinités orientales à Herculanum, p. 42 et pl. 27-28; A. ROULLET, The Egyptian and Egyptianizing Monuments of Imperial Rome, Leyde, 1972 (EPRO, 20), pl. XXIV, XXVIII; M. MALAISE, Inventaire préliminaire des documents égyptiens découverts en Italie, Leyde, 1972 $(E P R O, 21)$, pl. du frontispice.

45 Cf. Malaise, ibidem.

46 Cf. supra, n. 25. 
s'identifier à Isis, à une époque pour laquelle nous ne possédons aucune trace avérée de la pratique de l'initiation 47 , de l'aveu même de l'auteur (p. 73). Pour le domaine romain, il croit cependant pouvoir invoquer la discrétion dont les initiées, largement recrutées parmi les affranchies, se seraient entourées à une époque où les cultes isiaques faisaient l'objet de persécutions répétées. Néanmoins, trois reliefs d'affranchis datant d'environ 40 av. J.-C. (p. 98-101) représenteraient déjà des isiaques, et donc des initiées. En fait, ainsi qu'Eingartner le reconnaît, seul un des trois documents peut être réellement considéré comme nous livrant l'image d'une isiaque. Il s'agit d'un monument funéraire figurant une famille d'affranchis, un homme, une femme et une jeune fille, cette dernière étant caractérisée comme isiaque par le sistre et la patère qui l'entourent et par l'inscription qui la désigne comme USIA PRIMA $S A C(R O R U M)^{48}$ ISIDIS. On remarquera d'abord que le titre sacrorum Isidis ne doit pas s'appliquer aux initiés, car, comme l'a souligné Vidman, une inscription de Forum Popili distingue explicitement la dédicante, dite sacror(um) Isidis, des initiés, appelés telestini. En outre, si le portrait de l'homme et la coiffure de la femme permettent de situer le monument vers 40 av. J.-C., l'étude de H.G. Frenz, citée par Eingartner, a établi que le relief figurant la jeune-fille, tout comme le sistre, la patère et la partie de l'inscription qui l'identifie, ont été refaits plus tardivement, vers 40 ap. J.-C., à en juger par la coiffure. On ne trouverait donc en fait aucune trace inconographique d'isiaque sur les monuments funéraires des affranchies de l'époque républicaine. Il n'en reste pas moins vrai, comme l'établit Eingartner sur base de l'onomastique des inscriptions grecques et latines, que le milieu des affranchis a fourni une bonne part du contingent des isiaques durant la haute époque impériale.

Quelle est donc en définitive l'identité de nos isiaques optant pour une mode qui semble bien différer de celle des prêtresses et qu'il nous paraît dangereux de vouloir identifier systématiquement à des initiées?

47 Sur cette question, outre le renvoi à Vidman, il aurait fallu citer Y. GRANDJEAN, Une nouvelle arétalogie d'Isis à Maronée, Leyde, 1973 (EPRO, 26), p. 75-79, qui penche pour l'existence de rites initatiques isiaques dès l'époque hellénistique; et U. BIANCHI, Iside misterica. Quando?, in Studia A. Brelich, Rome, 1980, p. 9-36; M. MALAISE, Contenu et effets de l'initiation isiaque, in AC, 50 (1981), p. 484-486 qui ne partagent pas cette manière de voir.

48 On peut restituer $S A C(R O R U M)$ ou moins vraisemblablement $S A C$ (ERDOS), cf. L. VIDMAN, Isis und Sarapis bei den Griechen und Römern, Berlin, 1970 (RGVV, 29), p. 88 . 
Le sistre ${ }^{49}$ et la situle ${ }^{50}$ qu'elles empoignent ne peuvent vraiment nous éclairer si l'on admet qu'elles ont simplement emprunté ces attributs à l'image de leur divine patronne ${ }^{51}$. Néanmoins, le sistre et la situle faisaient partie des instruments réellement utilisés lors du culte. Ainsi, Apulée (XI, 10;12) place le sistre entre les mains des initiés et du grandprêtre, tandis que, sur une peinture d'Herculanum, un chœur de dévots des deux sexes participe à une cérémonie en agitant des sistres ${ }^{52}$. On peut dès lors se demander, vu le nombre assez élevé de ces isiaques en un lieu comme Athènes, si certaines femmes revêtues des atours d'Isis ne sont pas à identifier aux chanteuses et musiciennes auxquelles on recourait déjà dans la liturgie pharaonique ${ }^{53}$. Le statut des musiciennes sacrées attachées aux temples d'Égypte recouvrait des états différents: certaines faisaient partie du clergé proprement dit, d'autres excerçaient leurs talents à titre occasionnel. Dans le Décret trilingue de Canope ${ }^{54}$, il est question de jeunes-filles, désignées en grec comme

49 Cf. N. Genaille, Documents égyptisants du Musée des Antiquités de Turin, in RA (1975), II, p. 232-235; EAD., Le sistre Strozzi (à propos des objets cultuels isiaques en Italie), in Bull. de la Soc. Franç. d'Egyptologie, 77-78 (1976-77), p. 55-67; EAD., art. Sistrum, diffusion gréco-romaine, in Lexikon der Ägyptologie, V, 1984, col. 963-965. Pour l'Égypte, cf. Ch. ZIEGLER, art. Sistrum, in Lexikon der Ägyptologie, V, 1984, col. 959-963.

50 Cf. J.G. Griffiths, Apuleius of Madauros. The Isis-Book, p. 208-210.

51 Comme le souligne N. GENAILLE, in Lexikon der Ägyptologie, V, 1984, col. 963, «De fait, lors de la diffusion des cultes égyptiens dans le monde gréco-romain, le sistre devint l'insigne des divinités et officiants. Isis le portait, au lieu qu'il soit agité devant elle : ainsi la montrent les statues, ainsi elle apparaît en songe à Lucius, tenant aussi la situle libatoire; sans doute était-elle à l'origine considérée alors comme prêtresse de son propre culte».

52 Cf. V. Tran Tam TInh, Le culte d'Isis à Pompéi, p. 27 et pl. XXIII; ID., Le culte des divinités orientales à Herculanum, p. 43 et pl. XXVII. Il est vraisemblable que sur la seconde fresque d'Herculanum (ID., Pompéi, pl. XXIV et Herculanum, pl. XXVIII) les participants exécutent aussi des chants: cf. J.G. GRIFFITHS, Apuleius of Madauros. The Isis-Book, p. 186.

53 Cf. A.M. Blackman, On the Position of Women in the Ancient Egyptian Hierarchy, in Journal of Egyptian Archaeology, 7 (1921), p. 8-30; J. YOYOTTE, Les vierges consacrées d'Amon thébain, in CRAIBL, 1961, p. 43-52; F. Dunand, Le culte d'Isis dans le bassin oriental de la Méditerranée, I, p. 171 172; H.G. Fischer, art. Priesterin, in Lexikon der Ägyptologie, IV, 1982, col. 1100-1105. On songera aussi aux statues citées supra, n. 12.

54 Cf. K. SEThe, Urkunden, II, p. 150-151; Fr. DAUMAS, Les moyens d'expression du grec et de l'égyptien comparés dans les les déc, ets de Canope et de Memphis, Le Caire, 1952 (Suppl. aux Annales du Service des Antiquités de l'Eg., 16), p. 187. 
"vierges sacrées» (tôn hierôn parthenôn) et en égyptien comme "chanteuses» ( $\left.\zeta_{m} \cdot w t\right)$, qui, coiffées des couronnes des dieux dont elles sont les prêtresses (en grec : hiereiai; en égyptien : $w^{`} b . w t$ ), chanteront (en grec : hymneisthai; en égyptien : $d w 3$ ) en l'honneur de Bérénice. Le même décret nous apprend que d'autres chanteurs des deux sexes étaient requis pour le service quotidien et les fêtes de Bérénice; ils sont cette fois désignés comme "chanteurs, hommes et femmes" (en grec : ôidous andras kai gynaikas; en égyptien: $d m 3 . w h s . w t ~ t 3 j . w h m . w t$ ) et placés sous les ordres d'un maître de chœur (ôidodidaskalos). Ce texte nous paraît intéressant à plus d'un égard. D'abord, il montre bien l'importance de la musique et des chants ${ }^{55}$ dans le culte et l'existence, à côté de chanteuses sacrées, de musiciennes n'appartenant sans doute pas au clergé. On constate aussi que les chanteuses sacrées empruntent aux divinités, sinon leurs vêtements, du moins leurs couronnes. Quand enfin on sait que les chanteuses égyptiennes maniaient le sistre ${ }^{56}$ et que les cérémonies isiaques d'Herculanum se déroulaient avec le concours d'un chœur ${ }^{57}$ accompagné par le crépitement des mêmes instruments, il est tentant d'admettre que parmi les isiaques étudiées par Eingartner se trouvaient des chanteuses-musiciennes. Si toutes ces femmes prêtaient leur concours au culte isiaque, il est vraisemblable qu'elles ont pu le faire à des titres différents, soit comme des auxiliaires occasionnelles, soit comme initiées, et peut-être même comme prêtresses ${ }^{58}$.

55 Clément d'Alexandrie (Strom., VI, 4, 35, 3) place en tête de la procession qu'il décrit un chanteur, lequel fait partie des ministres de rang supérieur: cf. $M$. Malaise, Les conditions de pénétration et de diffusion des cultes égyptiens en Italie, Leyde, 1972 (EPRO, 22), p. 114, 121.

56 Cf. Ch. ZIEGLER, art. Sistrum, in Lexikon der Ägyptologie, V, 1984, col. 960 et n. 33. On notera aussi que l'image d'un sistrophore constitue l'idéogramme ou le déterminatif de plusieurs termes en rapport avec la musique et les musiciens: cf. EAD., op. cit., col. 959.

57 D'autres exemples manifestent dans les cérémonies isiaques le recours au chant: Apulée (XI, 9) mentionne dans le cortège de Kenchrées la participation d'un choeur de jeunes gens entonnant un hymne (cf. J.G. GRIFFITHS, Apuleius of Madauros, Isis-Book, p. 185-186); ailleurs, il explique que la voix des fidèles annonģait chaque matin dans le temple d'Isis la première heure du jour (XI, 20), suivant un usage égyptien (cf. M. MALAISE, Les conditions de pénétration, p. 139). Pour d'autres informations sur le chant et la musique, cf. Malaise, op.cit., p. 121-123, 134 (où il est notamment question de l'ordre sacré des Péanistes, attachés à Rome au culte de Sérapis et des dieux augustes, et de la maîtresse de Tibulle (I, 3, 29-32) qui, deux fois par jour, les cheveux dénoués, chante les hymnes en l'honneur d'Isis).

58 On rappellera à cet égard deux inscriptions parallèles de Cirta, en Algérie, qui mentionnent une sistrata sacerdos, à moins qu'il ne faille lire sistratae sacerdos, 
Ces isiaques ont dû cependant assez rarement exercer un véritable sacerdoce, car des prêtresses n'auraient pas manqué de mentionner cette fonction honorifique dans leurs inscriptions. Lorsque les reliefs funéraires s'ornent de la ciste mystique $\left(n^{\circ} 106,112,120,127,130\right)$, nous croirions volontiers, avec Eingartner, qu'ils émanent d'isiaques initiées. Pour appuyer son interprétation, l'auteur aurait dû rappeler que, selon Tibulle (I, 7, 48), la corbeille renfermait les objets sacrés tenus secrets (occultis conscia cista sacris), témoignage que corrobore Apulée (XI, 11) quand il parle de "la corbeille contenant ce qu'on dérobe aux yeux et recelant dans ses flancs les secrets de la sublime religion" 59 .

le terme sistrata s'appliquant alors à Isis : cf. J.-Cl. GRENIER, L'autel funéraire isiaque de Fabia Stratonice, Leyde, 1978 (EPRO, 71), p. 12-13, n. 30.

59 Sur le contenu de cette ciste, voir notre étude Ciste et hydrie, symboles isiaques de la puissance et de la présence d'Osiris, in J. RIEs (éd.), Le symbolisme dans les grandes religions. Actes du colloque de Louvain-la-Neuve 4-5 octobre 1983, Louvain-la-Neuve, 1985 (Homo Religiosus, 11), p. 135-143. 